\title{
Risk factors for mortality in ICU patients with Acinetobacter baumannii ventilator-associated pneumonia: impact of bacterial cytotoxicity
}

\author{
Mohan Ju" ${ }^{1 \#}$, Dongni Hou ${ }^{1 \#}$, Shu Chen ${ }^{2 \#}$, Ying Wang ${ }^{1}$, Xinjun Tang ${ }^{1}$, Jie Liu ${ }^{1}$, Cuicui Chen ${ }^{1}$, Yuanlin \\ Song ${ }^{1}$, Huayin $\mathrm{Li}^{1}$ \\ ${ }^{1}$ Department of Pulmonary Medicine, Zhongshan Hospital, Fudan University, Shanghai 200032, China; ${ }^{2}$ Department of Infectious Disease, Huashan \\ Hospital, Fudan University, Shanghai 200040, China \\ Contributions: (I) Conception and design: Y Song, H Li, M Ju; (II) Administrative support: None; (III) Provision of study materials or patients: M Ju; \\ (IV) Collection and assembly of data: M Ju, D Hou, Y Wang; (V) Data analysis and interpretation: M Ju, D Hou, Y Wang; (VI) Manuscript writing: \\ All authors; (VII) Final approval of manuscript: All authors. \\ "These authors contributed equally to this work. \\ Correspondence to: Yuanlin Song, MD; Huayin Li, MD. Department of Pulmonary Medicine, Zhongshan Hospital, Fudan University, 180 Fenglin \\ Road, Shanghai 200032, China. Email: ylsong70@163.com or song.yuanlin@zs-hosptial.sh.cn; li.huayin@zs-hosptial.sh.cn.
}

Background: Acinetobacter baumannii (A. baumannii) ventilator-associated pneumonia (VAP) in intensive care unit $(\mathrm{ICU})$ is associated with high morbidity and mortality in patients with critical illness. However, the literatures that focused on the short-term prognosis and the risk factors for mortality are limited. The aim of this study was to evaluate the risk factors for mortality in ICU patients with A. baumannii VAP.

Methods: A retrospective cohort study was conducted in the medical/surgical ICU at Zhongshan Hospital in Shanghai, China. Adult patients meeting the criteria of A. baumannii VAP from January 2012 to October 2015 were enrolled. Apart from collecting clinical and microbiologic data, we performed biofilm-formation and cytotoxicity testing using $A$. baumannii strains which are isolated from patients. Multivariate logistic regression analysis was used to determine the independent risk factors for 30-day mortality in ICU.

Results: Seventy-eight patients were included in this study. The 30-day mortality rate in ICU for the patients was $37.2 \%$. Multivariate analysis revealed that short-term mortality was significantly associated with prior surgery [OR, 0.277; 95\% confidence interval (CI), 0.089-0.866; $\mathrm{P}=0.027$ ], higher APACHEII score (OR, 1.140; 95\% CI, 1.007-1.291; $\mathrm{P}=0.038)$ and an increased bacterial cytotoxicity (OR, 1.029 ; $95 \% \mathrm{CI}$, 1.001-1.058; $\mathrm{P}=0.047)$.

Conclusions: The main finding of our study was that increased bacterial cytotoxicity might be a risk factor for short-term mortality in ICU patients with A. baumannii VAP.

Keywords: Acinetobacter baumannii (A. baumannii); cytotoxicity; intensive care unit; ventilator-associated pneumonia (VAP); prognosis

Submitted Sep 28, 2017. Accepted for publication Mar 13, 2018.

doi: $10.21037 /$ jtd.2018.04.86

View this article at: http://dx.doi.org/10.21037/jtd.2018.04.86 


\section{Introduction}

Ventilator-associated pneumonia (VAP) is one of the most common nosocomial infections in patients who receive mechanical ventilation. The occurrence of VAP prolongs stays in the intensive care unit (ICU) and hospital and is also associated with higher costs and a poorer prognosis (1-3).

Despite substantial clinical efforts, the incidence and mortality of VAP have remained high, with two recent epidemiologic studies in China reporting mortality rates of $18.7 \%$ and $26.7 \%(4,5)$.

Acinetobacter baumannii (A. baumannii) is showing an increasingly important role in nosocomial infection and it is thought to be the prevailing pathogen responsible for VAP in ICUs $(6,7)$ with high mortality (8-10). Since A. baumannii is an opportunistic pathogen, its direct virulence to host cells is thought to be minimum to moderate, but high virulence A. baumannii has been isolated in a recent report (11). Whether the cytotoxicity of $A$. baumannii affects patient prognosis remains unclear. In the present study, we aimed to identify risk factors for 30-day mortality in ICU patients with A. baumannii VAP considering both bacterial (biofilm formation and cytotoxicity) and patient characteristics.

\section{Methods}

\section{Study design}

A retrospective study was conducted in Zhongshan Hospital of Fudan University in Shanghai. All adult patients who were admitted to the medical/surgical ICU of Zhongshan Hospital between January2012 and October 2015 diagnosed with $A$. baumannii VAP according to 2005 ATS/IDSA criteria (12) were included. The study was reviewed and approved by the Clinical Research Ethics Committee of Zhongshan Hospital [2011212], Fudan University.

\section{Patients}

The criteria used for the diagnosis of VAP were the presence of a new or progressive radiographic infiltrate, consolidation, cavitation or pleural effusion in a patient undergoing mechanical ventilation, plus at least 2 of the following: (I) temperature $>38.3$ or $<36^{\circ} \mathrm{C}$; (II) purulent tracheal secretions or a change in sputum characteristics, and (III) white blood cell count $>10,000$ or $<4,000$ cells $/ \mathrm{mm}^{3}$.

Tracheal aspirate culture was used for the pathogenic diagnosis of VAP. Only the first tracheal aspirate episode from each patient was included. Pathogens meeting the diagnostic threshold for semi-quantitative culture were thought to be the cause of VAP. Semi-quantitative culture results can be classified into four categories: $+=$ virtually no growth, $++=$ little growth, $+++=$ moderate growth, and ++++ $=$ substantial growth. Results $>++$ were deemed positive (13).

\section{Clinical data collection}

All patients who met the clinical and microbiological diagnosis of $A$. baumannii VAP were analyzed. The following data were recorded: age, sex, length of ICU stay, ICU days before VAP, mechanical ventilation days before VAP, VAP classification, surgery within 30 days before diagnosis, disease severity at the time of diagnosis, comorbid conditions, invasive procedures, medical imaging features, appearance of subsequent $A$. baumannii bacteremia , antimicrobial susceptibility of $A$. baumannii, prior antibiotics and appropriateness of antibiotic therapy after diagnosis.

VAP was classified as either early onset $(<5$ days within mechanical ventilation) or late onset ( $\geq 5$ days). Disease severity was assessed using the Acute Physiology and Chronic Health Evaluation II (APACHEII) score upon diagnosis. Comorbid conditions were divided into groups as follows: diabetes mellitus, cardiovascular disease (coronary artery disease, cardio-myopathy and valvular heart disease), malignancy (hematologic malignancy and solid tumors), COPD (chronic obstructive pulmonary disease), liver cirrhosis, chronic kidney disease, cerebrovascular accident (cerebral infarction or cerebral hemorrhage) and transplant. Invasive procedures included tracheostomy, nasogastric tube placement and central venous catheterization. Medical imaging features included the presence of bilateral lung involvement and pleural effusion. Subsequent bacteremia was defined as at least one $A$. baumannii positive blood culture in the absence of a different infection source and at least one bacteria positive tracheal aspirate culture after the development of newly diagnosed AbVAP. Prior antibiotics treatment was defined as the use of systemic antibiotics for at least 72 hours within the preceding 14 days before diagnosis. Appropriate antibiotic therapy was defined as the receipt of one or more antimicrobial agents to which $A$. baumannii was susceptible via an appropriate route within 48 hours of diagnosis.

\section{Antimicrobial susceptibility}

Susceptibility testing of 13 antimicrobials was performed by using the broth microdilution method. Susceptibility 


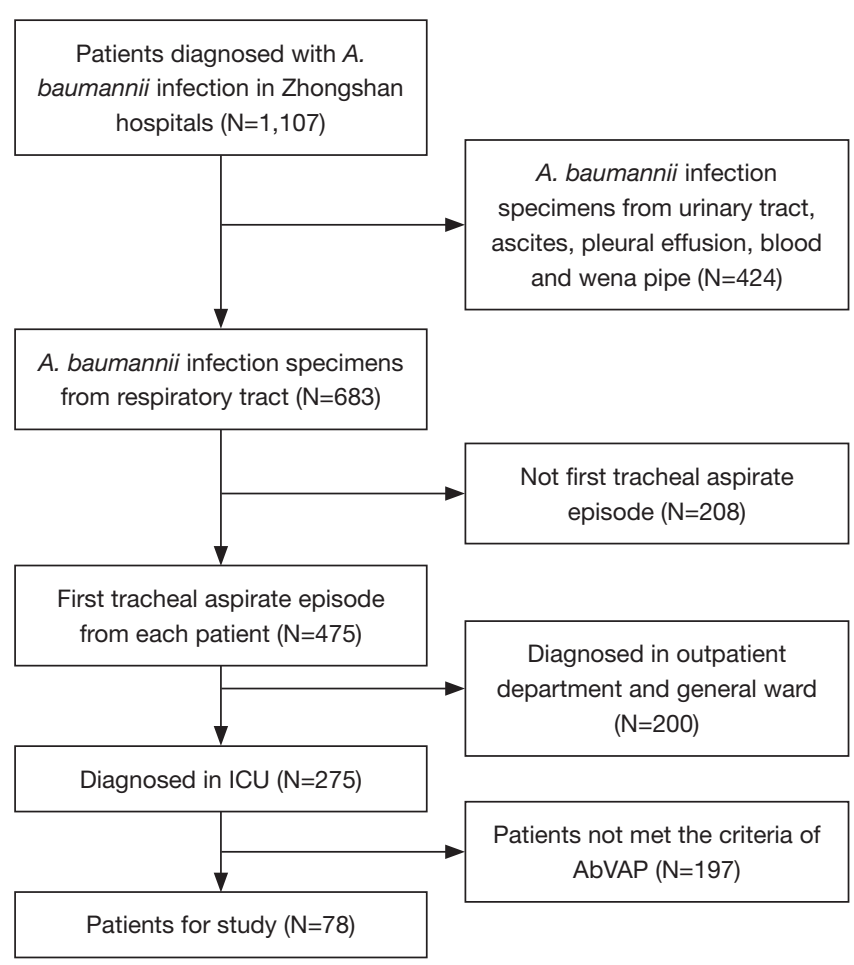

Figure 1 Patient selection.

results were interpreted according to guidelines established by the Clinical and Laboratory Standards Institute (14). Interpretation break points for tigecycline of $\leq 1 \mathrm{mg} / \mathrm{L}$ were considered as susceptible, and $\geq 4 \mathrm{mg} / \mathrm{L}$ as resistant according to European Committee on Antimicrobial Susceptibility Testing (EUCAST) criteria (15).

\section{Biofilm-formation}

Biofilm formation was tested as follows (16): single $A$. baumannii colonies were cultured in Luria-Bertani broth (LB broth) with shaking at $37^{\circ} \mathrm{C}$ for 16 hours and then diluted with LB broth to a McFarland turbidity of 0.5 . The suspensions were deposited in 96-well microtiter plates and incubated for $48 \mathrm{~h}$. Each sample had two duplicates, and the negative control was LB broth. After incubation, phosphatebuffered solution was used to clean the wells, and methanol was used as a fixative. Biofilms were stained with $200 \mu \mathrm{L}$ of $0.1 \%$ crystal violet solution for $20 \mathrm{~min}$. After that, the wells were washed with slow flowing water three times, and the remaining stain was solubilized with $160 \mu \mathrm{L}$ of $33 \%$ glacial acetic acid solution. Finally, biofilm biomass was measured by determining the absorbance at $570 \mathrm{~nm}\left(\mathrm{OD}_{570}\right)$. a $\mathrm{OD}_{570}$ $>0.7$ is considered a biofilm production.

\section{Cytotoxicity}

Cytotoxicity assessment was tested as follows $(17,18)$ : A. baumannii strains were cultured in tryptone broth at $37^{\circ} \mathrm{C}$ with shaking at $200 \mathrm{rpm}$ overnight. Small-quantity suspensions were cultured again in new medium for $4 \mathrm{~h}$ when reaching the exponential phase. The bacterial suspensions were then collected and centrifuged for 5 minutes at $10,000 \mathrm{rpm}$ and adjusted to $1.0 \times 10^{8} \mathrm{cfu} / \mathrm{mL}$ with phosphate buffered saline (PBS). Immortalized human bronchial epithelial cells (BEAS-2B cells) were seeded in 96-well plates at a density of $2.5 \times 10^{4}$ cells/well and cultured overnight the day before the experiment. Each well of cells was infected with an $A$. baumannii suspension at a multiplicity of infection (MOF) of $200: 1$. Infected plates were then incubated at $37{ }^{\circ} \mathrm{C}$ with $5 \% \mathrm{CO}_{2}$ for 2 hours. Each bacterial sample had two duplicates. Cytotoxicity was measured using a Cytotoxicity LDH Assay KitWST (Dojindo Kumamoto, Japan, CK12) following the manufacturer's protocol.

\section{Follow-up and outcomes}

Overall 30-day mortality in ICU was the outcome. All patients were followed up for survival status until death or 30 days after onset of VAP.

\section{Statistical analysis}

Data were processed with SPSS for Windows (Version 22.0, SPSS Inc., Chicago, IL, USA). Continuous variables were analyzed using Student's $t$-test or the Mann-Whitney $\mathrm{U}$ test, whereas categorical variables were analyzed using the chi-squared test or Fisher's exact test. Risk factors for 30-day mortality were identified using logistic regression (all clinical and laboratory parameters were analyzed in a multivariate logistic regression model). $\mathrm{P}<0.05$ were considered statistically significant.

\section{Results}

\section{Clinical data at presentation}

After selection, a total of 78 patients diagnosed with $A$. baumannii VAP in ICU were included in this study (Figure 1). According to Table 1, the mean patient age was $58.8 \pm 12.8$ years old, $58(74.4 \%)$ of the patients were male, 54 patients had early-onset VAP and $69.2 \%$ of the patients underwent recent surgery (within 30 days). The average 
Table 1 Demographic and clinical characteristics of patients with $A$. baumannii ventilator-associated pneumonia

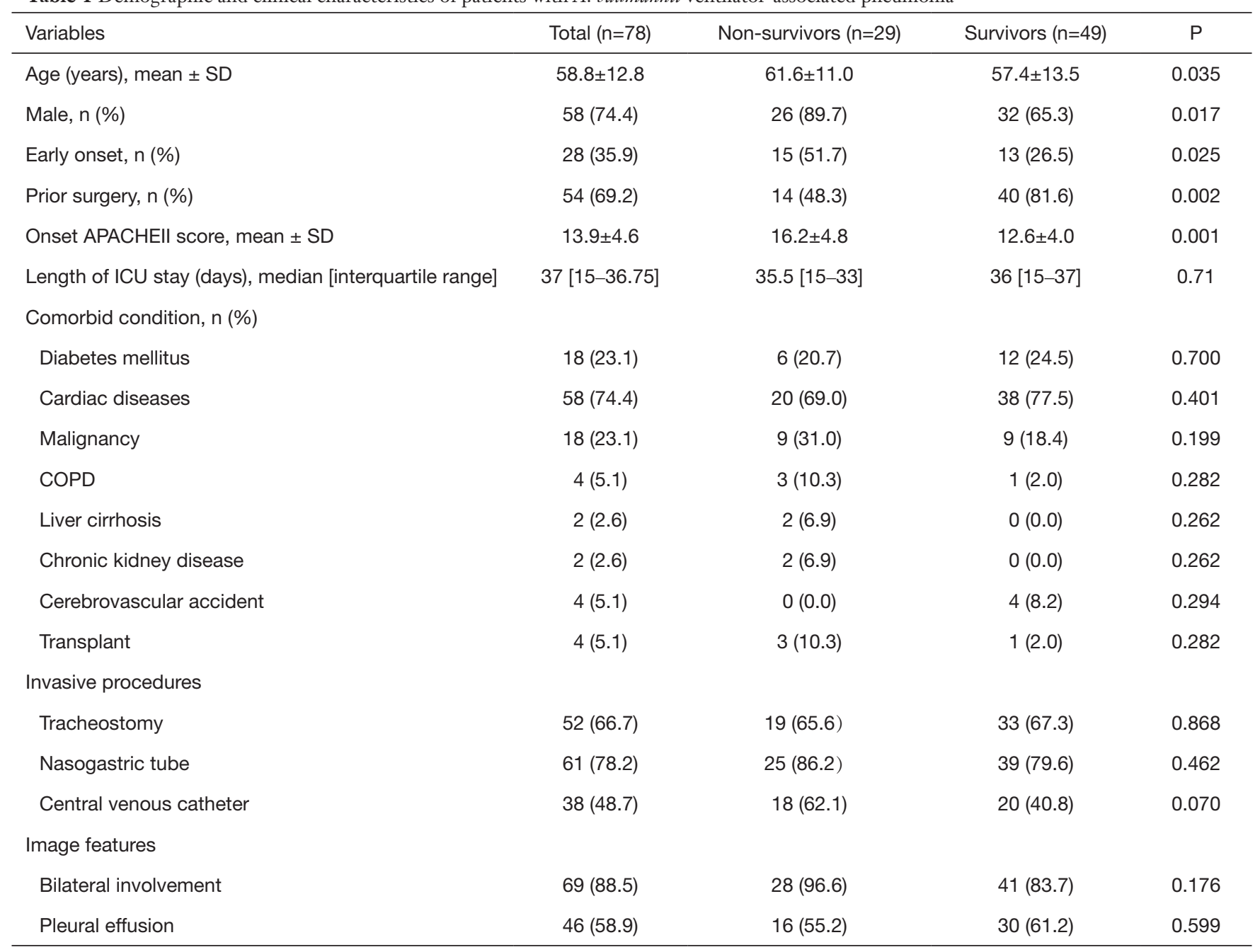

Early onset VAP is defined as VAP occurring $<5$ days within mechanical ventilation. Prior surgery is defined as surgery within 30 days before diagnosis; APACHEII, Acute Physiology and Chronic Health Evaluation II; Cardiovascular disease includes coronary artery disease, cardio-myopathy and valvular heart disease; Malignancy includes hematologic malignancies and solid tumor; COPD, chronic obstructive pulmonary disease; cerebrovascular accident includes cerebral infarction or cerebral hemorrhage.

APACHEII score at the time of diagnosis was $13.9 \pm 4$.6. Cardiovascular disease was the most common underlying systemic disease and was found in $74.4 \%$ of the patients. The most common invasive procedure performed during the ICU stay was nasogastric tube placement $(61,78.2 \%)$. According to chest radiography, $88.5 \%$ of the patients had bilateral lung involvement, and $58.9 \%$ of the patients had pleural effusion.

\section{Pathogen characteristics and antibiotic therapy}

Susceptibility profiles of tracheal aspirate isolates are presented in Table 2. All these A. baumannii isolates only showed sufficient susceptibility to colistin $(78,100 \%)$ and tigecycline (61, 78.2\%). Carbapenem-resistant $A$. baumannii VAP accounted for $79.5 \%$ of the cases. Overall, only $37.2 \%$ of the patients received appropriate antibiotic therapy. In the present study, we found that the biofilm-production rate was significantly higher in survivors than in non-survivors [40.8\% (20/49) vs. $13.8 \%$ (4/29), $\mathrm{P}=0.012]$ and we also revealed the non-survivor group exhibited higher bacterial cytotoxicity than the survivor group $(65.1 \% \pm 24.2 \% v s$. $51.4 \% \pm 15.0 \%, \mathrm{P}=0.009)$. 
Table 2 Pathogen characteristics and antibiotic therapy of patients with $A$. baumannii ventilator-associated pneumonia

\begin{tabular}{|c|c|c|c|c|}
\hline Variables & Total $(\mathrm{n}=78)$ & Non-survivors $(\mathrm{n}=29)$ & Survivors $(n=49)$ & $\mathrm{P}$ \\
\hline Amikacin resistance & $59(75.6)$ & $23(79.3)$ & $36(73.5)$ & 0.561 \\
\hline Ceftazidime resistance & $65(83.3)$ & $25(86.2)$ & $40(81.6)$ & 0.834 \\
\hline Ciprofloxacin resistance & $65(83.3)$ & $25(86.2)$ & $40(81.6)$ & 0.834 \\
\hline Cefepime resistance & $65(83.3)$ & $25(86.2)$ & $40(81.6)$ & 0.834 \\
\hline Gentamycin resistance & $67(85.9)$ & $26(89.7)$ & $41(83.7)$ & 0.691 \\
\hline Meropenem resistance & $62(79.5)$ & $23(79.3)$ & $39(79.6)$ & 0.976 \\
\hline SMZ-TZP resistance & $56(71.8)$ & $20(69.0)$ & $36(73.5)$ & 0.669 \\
\hline Piperacillin/tazobactam resistance & $65(83.3)$ & $25(86.2)$ & $40(81.6)$ & 0.834 \\
\hline Tigecycline resistance & $17(21.8)$ & $6(20.7)$ & $11(22.4)$ & 0.856 \\
\hline Colistin resistance & $0(0)$ & $0(0)$ & $0(0)$ & - \\
\hline Subsequent $A$. baumannii bacteremia, n (\%) & $6(7.7)$ & $4(13.8)$ & $2(4.1)$ & 0.264 \\
\hline \multicolumn{5}{|l|}{ Treatment } \\
\hline Inappropriate antibiotic therapy, n (\%) & $49(62.8)$ & $23(79.3)$ & $26(53.1)$ & 0.020 \\
\hline Biofilm production, n (\%) & $24(30.8)$ & $4(13.8)$ & $20(40.8)$ & 0.012 \\
\hline \multicolumn{5}{|l|}{ Cytotoxicity } \\
\hline
\end{tabular}

SMZ-TZP, sulfamethoxazole-trimethoprim; LDH, lactic dehydrogenase.

Outcomes and prognostic factors for 30-day mortality of $A$. baumannii VAP in ICU

The 30-day mortality rate in ICU for 78 patients was $37.2 \%$ (29/78). The results of univariate analysis demonstrated that age, gender, early-onset VAP, prior surgery within 30 days before diagnosis, APACHEII score at the time of diagnosis, inappropriate antibiotic therapy, biofilm production and high LDH releasing ratio which is representative of cytotoxicity were associated with higher 30-day mortality rates in patients with $A$. baumannii VAP.

For logistic regression analysis, with only 29 deceased patients, the number of co-variates added into the regression model can at most be 3 , these included prior surgery, APACHEII score and LDH release ratio. As shown in Table 3, prior surgery [OR, $0.277 ; 95 \%$ confidence interval (CI), 0.089-0.866; $\mathrm{P}=0.027$ ], higher APACHII score (OR, $1.140 ; 95 \%$ CI, $1.007-1.291 ; \mathrm{P}=0.038)$ and an increased bacterial cytotoxicity (OR, 1.029 ; 95\% CI, 1.001-1.058; $\mathrm{P}=0.047$ ) were identified as independent prognostic factors for 30-day ICU mortality of $A$. baumannii VAP patients.

\section{Characteristics of A. baumannii VAP patients with high/ low cytotoxic bacterial infection}

In this context, owing to no widely accepted cutpoint of LDH releasing ratio in $A$. baumannii species, we used the average value of $56.5 \%$ as the cutoff for dichotomization. The characteristics of patients with high/low cytotoxic bacterial infection were compared in Table 4, showing that the patients with high cytotoxic bacterial infection had higher APACHEII score at the time of diagnosis. Susceptibility testing results also indicated a higher antibiotics resistance rate to most kind of antibiotics in patient with low cytotoxic bacterial infection. Kaplan-Meier 
Table 3 Multivariate logistic regression analysis for predictors of mortality in patients $A$. baumannii ventilator-associated pneumonia

\begin{tabular}{lccc}
\hline Variable & $\mathrm{P}$ & $\mathrm{OR}$ & $95 \% \mathrm{Cl}$ \\
\hline Prior surgery & 0.027 & 0.277 & $0.089-0.866$ \\
APACHEll score & 0.038 & 1.140 & $1.007-1.291$ \\
LDH releasing ratio (\%) & 0.047 & 1.029 & $1.001-1.058$ \\
\hline
\end{tabular}

$\mathrm{LDH}$, lactic dehydrogenase.

analysis showed high bacterial cytotoxicity can significantly affect the short-term prognosis of patents (Figure 2).

\section{Discussion}

The present study had revealed that high bacterial cytotoxicity was independent risk factor for mortality in patients with $A$. baumannii VAP. Prior to our study, the impact of bacterial cytotoxicity on the prognosis of $A$. baumannii VAP in ICU was still not clear, especially in Chinese patients.

At the end of follow-up, 29 patients died (30-day mortality rate: $37.2 \%$ ), which was comparable to the study by Inchai et al. (30-day mortality rate: 48.3\%) (19), Haliloglu et al. (28-day mortality rate: $52.3 \%$ ) (20), and Tsioutis et al. (28-day mortality rate: 29.8\%) (21). Thus, no obvious difference in the mortality rate was found between our study and the previous studies.

Higher APACHEII score at diagnosis were found in nonsurvivor group than in survivor group, which is consistent with previous researches on $A$. baumannii bloodstream infection $(22,23)$. Interestingly, however, recent surgery acted as a protective factor, which may due to better organ function in patients who could tolerate surgery.

Numerous and diverse underlying systemic diseases have been reported as potential risk factors for mortality in $A$. baumannii infection (19-24). These include malignancy, post-transplantation status and chronic respiratory disease following previous survey. In the current study, we failed to identify a significantly higher mortality rate in patients with a certain kind of comorbid condition.

The presence of $A$. baumannii bacteremia always meant that the patients were in a state of severe illness. It is well accepted that VAP patient in the ICU often develop subsequent bacteremia, which were associated with a poorer outcome. Brotfain et al. (25) demonstrated that ICU mortality rate was higher in patients with $A$. baumannii VAP having a secondary $A$. baumannii bacteremia compared to nonbacteremic patients. Six $(7.7 \%)$ patients had a subsequent $A$. baumannii bacteremia in our study, and after multivariate analysis, subsequent bacteremia was not found to be an independent predictor for short-term ICU mortality in patients with $A$. baumannii VAP, which is not accord with the conclusion of research by Magret $e t$ al. (26). This may be explained by the small sample size of our single-center study.

The main finding of our study was that high cytotoxicity was an independent risk factor significantly related to mortality in patients with $A$. baumannii VAP. Lactic dehydrogenase $(\mathrm{LDH})$ is a stable cytoplasmic enzyme presented in all types of cells and released into the cell culture medium through damaged plasma membrane. In the present study, we used immortalized human bronchial epithelial cells (BEAS-2B cells) as a model system to assess $A$. baumannii cytotoxicity in vitro by measuring the released LDH according to Cytotoxicity LDH Assay Kit-WST. As shown in Table 2, the non-survivor group exhibited higher bacterial cytotoxicity than the survivor group. The results from multivariate analysis also showed that $A$. baumannii cytotoxicity is associated with poorer outcomes in $A$. baumannii VAP patients. This result is inconsistent with a previous study by Lázaro-Díez et al. (27), in which $A$. baumannii strains failed to induce any cytotoxic effect on lung epithelial cells. However, the referenced study examined only a handful of clinical $A$. baumannii strains (5 in total) and used the human lung epithelial A549 cell line as a model system to measure cytotoxicity with double immunofluorescence labeling and confocal microscopy. In contrast, we used BEAS-2B cells and the LDH-release method to examine cytotoxicity. Furthermore, the aim of our study was to identify whether an association exists between $A$. baumannii cytotoxicity and patient prognosis. The mechanisms of $A$. baumannii cytotoxicity have been previously studied. For example, Choi et al. (28) reported that the membrane proteins found in A. baumannii induce epithelial cell apoptosis by signaling through cell surface death receptors and mitochondrial targeting. Lipopolysaccharides (LPS) present in the outer membranes of bacterial so induce innate immune responses in a Tolllike receptor4 (TLR4)-dependent manner. Lin et al. (29) found that $\mathrm{LpxC}$ inhibitors affect the biosynthesis of lipid A, a core immune molecule induced by LPS that can enhance opsonophagocytic killing and thereby reduce the mortality of infected mice. We are presently working on isolating cytotoxic genes from strains with high $\mathrm{LDH}$ release to screen for potential predictive biomarkers and 
Table 4 Comparison of the clinical characteristics of patients with high or low cytotoxicity bacterial infection

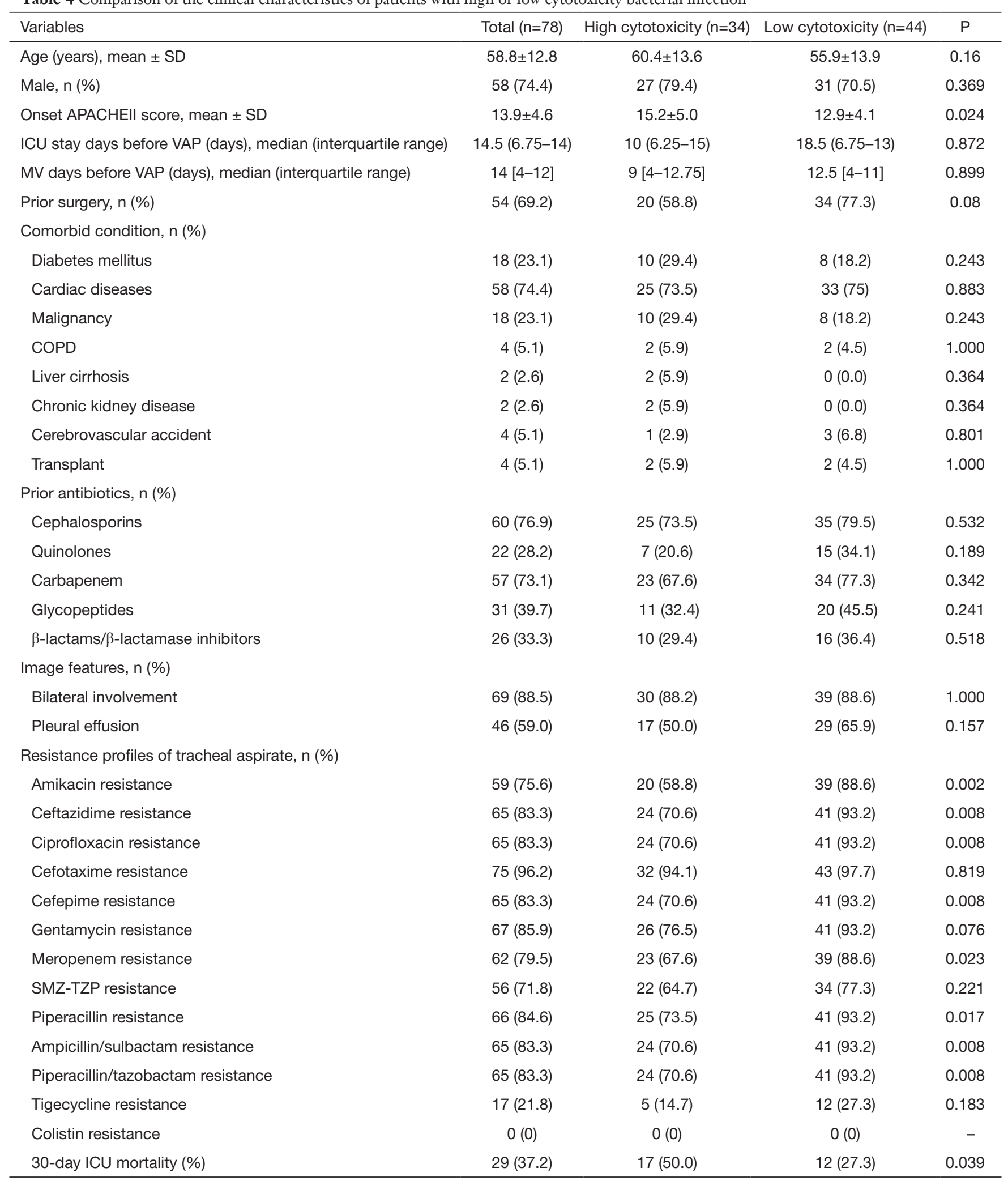

VAP, ventilator-associated pneumonia; ICU, intensive care unit. 


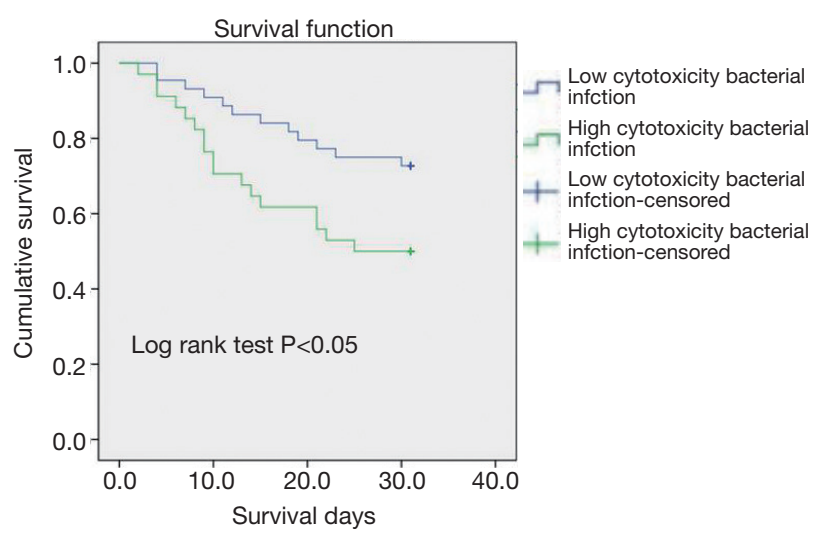

Figure 2 Kaplan-Meier analysis for patients with high or low cytotoxicity bacterial infection. We used 30-day ICU mortality as the main outcome for assessment of mortality for patients with serious conditions due to A. baumannii ventilator associated pneumonia. High bacterial cytotoxicity can significantly affect the short-term prognosis of patents (log rank test $\mathrm{P}=0.029)$.

identify new targets for clinical treatment.

A higher antibiotics resistance rate to most kind of antibiotics in patient with low cytotoxic bacterial infection was found in this survey. Many researchers studied the link between antimicrobial resistance and virulence and found some controversial result. For example, MexEF-OprN efflux pump is an important system for $P$. aeruginosa to extrude antibiotics but it also takes part in the downregulation of bacterial pathogenicity through a gene mutation that affect the cell quorum-sensing system (30); inactivation of MuxABC-OpmB transporter system in $P$. aeruginosa leads to increased ampicillin and carbenicillin resistance and decreased virulence due to decreased twitching motility (31). Further studies on A. baumannii are needed.

Previous studies have shown that administering adequate antibiotic therapy is crucial to a good therapeutic outcome $(32,33)$. Appropriate antibiotic therapy was defined as the administration of antibiotics to which $A$. baumannii was susceptible through an appropriate route within 48hours of diagnosis in this study. Our data demonstrate that early administration of appropriate antimicrobial treatment improved the outcomes of patients with $A$. baumannii VAP. However, $62.8 \%$ of the patients did not receive appropriate treatment upon diagnosis, and this number increased to $79.3 \%$ in the non-survivor group. This result suggests that emphasis should be paid on testing drug sensitivity to avoid inappropriate therapy.

A. baumannii has been associated with VAP, bacteremia, meningitis, urinary tract infections and wound infections in hospitals partly because of its emerging antimicrobial resistance, especially carbapenem resistance. There have been reports on the factors associated with antibiotic resistance-related mortality (34-36). We found carbapenem resistance rate reach almost $80 \%$ in our study, while carbapenem resistance was not an independent prognostic factor for 30-day ICU mortality in patients. The difficulty in treating carbapenem-resistant $A$. baumannii infections is still notable, tigecycline may play an important role in alternative antibiotic therapy: most of carbapenem-resistant A. baumannii VAP patients receive tigecycline treatment after diagnosis in our study. Since colistin represents a last resort drug for MDR gram negative pathogens, its access to mainland China can be a great help for patients with $A$. baumannii infection.

Limitations were inherent in this study, all clinical information with reference to risk factors was retrospectively collected at a single hospital, which restricted the generalization of our result to more patients. We also do not have data on antimicrobial concentrations and about rapid worsening medical imaging. In addition, only 78 strains were used to assess biofilm production and cytotoxicity in vitro. Further prospective studies should be conducted to confirm the results.

\section{Conclusions}

In conclusion, based on the present study and to our knowledge, bacterial cytotoxicity was firstly proposed as an independent risk factor for mortality in patients with $A$. baumannii VAP. More attention should be paid in VAP not only on patients clinical but also on bacterial characteristics such as cytotoxicity. Exact mechanisms of cytotoxicity in patients need to be investigated. Validating the current findings in a large prospective cohort study in future is also necessary.

\section{Acknowledgements}

The authors would like to thank Sarah Conte for her linguistic assistance with the manuscript.

Funding: This research was supported by the National Natural Science Foundation of China (81500026, 81400043, 81770055, 81630001, 81490533). Dr. Y Song was supported by the State Key Basic Research Program (973) project (2015CB553404) and Shanghai Science and Technology Committee Grant (No. 15DZ1930600, No. 15DZ1930602). 


\section{Footnote}

Conflicts of Interest: The authors have no conflicts of interest to declare.

Ethical Statement: The study was reviewed and approved by the Clinical Research Ethics Committee of Zhongshan Hospital (2011212), Fudan University.

\section{References}

1. Craven DE, Lei Y, Ruthazer R, et al. Incidence and outcomes of ventilator-associated tracheobronchitis and pneumonia. Am J Med 2013;126:542-9.

2. Kollef MH, Hamilton CW, Ernst FR. Economic impact of ventilator-associated pneumonia in a large matched cohort. Infect Control Hosp Epidemiol 2012;33:250-6.

3. Mathai AS, Phillips A, Kaur P, et al. Incidence and attributable costs of ventilator-associated pneumonia (VAP) in a tertiary-level intensive care unit (ICU) in northern India. J Infect Public Health 2015;8:127-35.

4. Zhou Q, Lee SK, Jiang S, et al. Efficacy of an infection control program in reducing ventilator-associated pneumonia in a Chinese neonatal intensive care unit. Am J Infect Control 2013;41:1059-64.

5. Song X, Chen Y, Li X. Differences in incidence and outcome of ventilator-associated pneumonia in surgical and medical ICUs in a tertiary hospital in China. Clin Respir J 2014;8:262-8.

6. El-Saed A, Balkhy HH, Al-Dorzi HM, et al. Acinetobacter is the most common pathogen associated with late-onset and recurrent ventilator-associated pneumonia in an adult intensive care unit in Saudi Arabia. Int J Infect Dis 2013;17:e696-701.

7. Nhu NT, Lan NP, Campbell JI, et al. Emergence of carbapenem-resistant Acinetobacter baumannii as the major cause of ventilator-associated pneumonia in intensive care unit patients at an infectious disease hospital in southern Vietnam. J Med Microbiol 2014;63:1386-94.

8. Kalin G, Alp E, Akin A, et al. Comparison of colistin and colistin/sulbactam for the treatment of multidrug resistant Acinetobacter baumannii ventilator-associated pneumonia. Infection 2014;42:37-42.

9. Zalts R, Neuberger A, Hussein K, et al. Treatment of carbapenem-resistant Acinetobacter baumannii ventilatorassociated pneumonia: retrospective comparison between intravenous colistin and intravenous ampicillin-sulbactam. Am J Ther 2016;23:e78-85.
10. Özgür ES, Horasan ES, Karaca K, et al. Ventilatorassociated pneumonia due to extensive drug-resistant Acinetobacter baumannii: risk factors, clinical features, and outcomes. Am J Infect Control 2014;42:206-8.

11. Jones CL, Clancy M, Honnold C, et al. Fatal outbreak of an emerging clone of extensively drug-resistant Acinetobacter baumannii with enhanced virulence. Clin Infect Dis 2015;61:145-54.

12. American Thoracic Society, Infectious Diseases Society of America. Guidelines for the management of adults with hospital-acquired, ventilator-associated, and healthcareassociated pneumonia. Am J Respir Crit Care Med 2005;171:388-416.

13. Hashimoto S, Shime N. Evaluation of semi-quantitative scoring of Gram staining or semi-quantitative culture for the diagnosis of ventilator-associated pneumonia: a retrospective comparison with quantitative culture. J Intensive Care 2013;1:2.

14. Franklin R, Cockerill MD 3rd. Performance Standards for Antimicrobial Susceptibility Testing, Twenty-First Informational Supplement M100-S21. Clinical and Laboratory Standard Institute 2011;31:68-80.

15. Kahlmeter G, Brown DFJ, Goldstein FW, et al. European Committee on Antimicrobial Susceptibility Testing (EUCAST) technical notes on antimicrobial susceptibility testing. Clin Microbiol Infect 2006;12:501-3.

16. Martí S, Rodríguez-Baño J, Catel-Ferreira $M$, et al. Biofilm formation at the solid-liquid and air-liquid interfaces by Acinetobacter species. BMC Res Notes 2011;4:5.

17. Cano V, Moranta D, Llobet-Brossa E, et al. Klebsiella pneumoniae triggers a cytotoxic effect on airway epithelial cells. BMC Microbiol 2009;9:156.

18. Wang Q, Li H, Zhou J, et al. PcrV antibody protects multidrug resistant Pseudomonas aeruginosa induced acute lung injury. Respir Physiol Neurobiol 2014;193:21-8.

19. Inchai J, Pothirat C, Bumroongkit C, et al. Prognostic factors associated with mortality of drug-resistant Acinetobacter baumannii ventilator-associated pneumonia. J Intensive Care 2015;3:9.

20. Haliloglu M, Bilgili B, Haliloglu O, et al. Vitamin D level is associated with mortality predictors in ventilatorassociated pneumonia caused by Acinetobacter baumannii. J Infect Dev Ctries 2016;10:567-74.

21. Tsioutis C, Kritsotakis EI, Karageorgos SA, et al. Clinical epidemiology, treatment and prognostic factors of extensively drug-resistant Acinetobacter baumannii ventilator-associated pneumonia in critically ill patients. 
Int J Antimicrob Agents 2016;48:492-7.

22. Park SY, Choo JW, Kwon SH, et al. Risk factors for mortality in patients with acinetobacter baumannii bacteremia. Infect Chemother 2013;45:325-30.

23. Zheng YL, Wan YF, Zhou LY, et al. Risk factors and mortality of patients with nosocomial carbapenemresistant Acinetobacter baumannii pneumonia. Am J Infect Control 2013;41:e59-63.

24. Liu Q, Li W, Du X, et al. Risk and prognostic factors for multidrug-resistant acinetobacter baumannii complex bacteremia: a retrospective study in a tertiary hospital of West China. PloS One 2015;10:e130701.

25. Brotfain E, Borer A, Koyfman L, et al. Multidrug resistance acinetobacter bacteremia secondary to ventilator-associated pneumonia: risk factors and outcome. J Intensive Care Med 2017;32:528-34.

26. Magret M, Lisboa T, Martin-Loeches I, et al. Bacteremia is an independent risk factor for mortality in nosocomial pneumonia: a prospective and observational multicenter study. Crit Care 2011;15:R62.

27. Lázaro-Díez M, Navascués-Lejarza T, Remuzgo-Martínez $\mathrm{S}$, et al. Acinetobacter baumannii and A. pittii clinical isolates lack adherence and cytotoxicity to lung epithelial cells in vitro. Microbes Infect 2016;18:559-64.

28. Choi CH, Hyun SH, Lee JY, et al. Acinetobacter baumannii outer membrane protein A targets the nucleus and induces cytotoxicity. Cell Microbiol 2008;10:309-19.

29. Lin L, Tan B, Pantapalangkoor P, et al. Inhibition of LpxC protects mice from resistant Acinetobacter baumannii by

Cite this article as: Ju M, Hou D, Chen S, Wang Y, Tang X, Liu J, Chen C, Song Y, Li H. Risk factors for mortality in ICU patients with Acinetobacter baumannii ventilator-associated pneumonia: impact of bacterial cytotoxicity. J Thorac Dis 2018;10(5):2608-2617. doi: 10.21037/jtd.2018.04.86 modulating inflammation and enhancing phagocytosis. MBio 2012;3:e00312-12.

30. Yang L, Chen L, Shen L, et al. Inactivation of MuxABCOpmB transporter system in Pseudomonas aeruginosa leads to increased ampicillin and carbenicillin resistance and decreased virulence. J Microbiol 2011;49:107-14.

31. Lamarche MG, Déziel E. MexEF-OprN efflux pump exports the Pseudomonas quinolone signal (PQS) precursor HHQ (4-hydroxy-2-heptylquinoline). PLoS One 2011;6:e24310.

32. Kim YJ, Kim SI, Hong KW, et al. Risk factors for mortality in patients with carbapenem-resistant Acinetobacter baumannii bacteremia: impact of appropriate antimicrobial therapy. J Korean Med Sci 2012;27:471-5.

33. Lee Y'T, Kuo SC, Yang SP, et al. Impact of appropriate antimicrobial therapy on mortality associated with Acinetobacter baumannii bacteremia: relation to severity of infection. Clin Infect Dis 2012;55:209-15.

34. Metan G, Sariguzel F, Sumerkan B. Factors influencing survival in patients with multi-drug-resistant Acinetobacter bacteraemia. Eur J Intern Med 2009;20:540-4.

35. Sheng WH, Liao CH, Lauderdale TL, et al. A multicenter study of risk factors and outcome of hospitalized patients with infections due to carbapenem-resistant Acinetobacter baumannii. Int J Infect Dis 2010;14:e764-9.

36. Huang ST, Chiang MC, Kuo SC, et al. Risk factors and clinical outcomes of patients with carbapenem-resistant Acinetobacter baumannii bacteremia. J Microbiol Immunol Infect 2012;45:356-62. 